\title{
Meta
}

Journal des traducteurs

Translators' Journal

\section{LE BLANC, Charles (2019) : Histoire naturelle de la traduction. Traductologiques. Paris : Les Belles Lettres, 304 p.}

\section{Jean Delisle}

Volume 64, numéro 3, décembre 2019

URI : https://id.erudit.org/iderudit/1070550ar

DOI : https://doi.org/10.7202/1070550ar

Aller au sommaire du numéro

Éditeur(s)

Les Presses de l’Université de Montréal

ISSN

0026-0452 (imprimé)

1492-1421 (numérique)

Découvrir la revue

Citer ce compte rendu

Delisle, J. (2019). Compte rendu de [LE Blanc, Charles (2019) : Histoire

naturelle de la traduction. Traductologiques. Paris : Les Belles Lettres, 304 p.]

Meta, 64(3), 845-847. https://doi.org/10.7202/1070550ar

Ce document est protégé par la loi sur le droit d'auteur. L'utilisation des services d'Érudit (y compris la reproduction) est assujettie à sa politique d'utilisation que vous pouvez consulter en ligne.

https://apropos.erudit.org/fr/usagers/politique-dutilisation/
Cet article est diffusé et préservé par Érudit.

Érudit est un consortium interuniversitaire sans but lucratif composé de l’Université de Montréal, l'Université Laval et l'Université du Québec à Montréal. Il a pour mission la promotion et la valorisation de la recherche. https://www.erudit.org/fr/ 
sujet a été fouillé, la recherche méticuleusement conduite, les conclusions, fondées sur une littérature savante et établie, peu discutables. On sort de sa lecture mieux au fait des difficultés inhérentes à la tâche de l'interprète judiciaire travaillant en contexte bilingue, quoique dans le cadre particulier qu'offre la situation épineuse de Hong Kong. Il reste que cette ancienne colonie (britannique), dotée d'institutions et de procédures judiciaires datant de l'ère coloniale et de la langue administrative du colonisateur, n'est pas un cas d'espèce, aussi symbolique et représentatif soit-il pour une personne qui - et ceci est le fait de l'humaine condition - regarde un tableau avec les yeux de Chimène. Les difficultés et obstacles qu'y rencontrent les interprètes judiciaires ne sont pas propres à Hong Kong, l'apanage d'un seul État; on les retrouve, en pire, dans nombre d'États parmi ceux qui ont accédé plus ou moins récemment à l'indépendance et doivent faire face à cette nouvelle situation, se projeter dans l'avenir à partir des sources, usages et «formants» légués par le colonisateur. On pourrait avancer le cas du Sri Lanka, celui du Cameroun ou, encore plus significatif, de l'Afrique du Sud, avec ses onze langues officielles - sans parler de l'Inde! On imagine sans peine les difficultés et les contraintes avec lesquelles ces systèmes judiciaires et leurs acteurs doivent composer. Les recommandations d'Eva N. S. Ng, dans ces cas-là, résonnent comme autant d'illusions lyriques. Il faut néanmoins lui savoir gré d'avoir effectué une telle recherche et produit un ouvrage de référence sur la situation difficile que vivent tant d'interprètes judiciaires dans les rapports qu'ils entretiennent avec l'autorité judiciaire et leur lutte incessante pour faire entendre et comprendre la parole des acteurs du procès.

Jean-Claude GÉmar Université de Montréal, Montréal, Canada

\section{RÉFÉRENCE}

NG, Eva (2016): Do they understand? English Trials Heard by Chinese jurors in the Hong Kong Courtroom. Language and Law/Linguagem e direito. 3(2):172-191.

LE BLANC, Charles (2019): Histoire naturelle de la traduction. Traductologiques. Paris: Les Belles Lettres, 304 p.

Il y a dix ans, lorsqu'est paru, du même auteur, Le complexe d'Hermès (Le Blanc 2009), ouvrage désormais accessible en anglais, en coréen, en arabe et en italien, j'avais dit dans une recension tout le bien que je pensais de cet essai sur la bonne manière de théoriser la pratique de la traduction (Delisle 2011).
L'auteur y diagnostiquait trois problèmes fondamentaux: la difficulté pour la traductologie de se constituer en discipline pleinement autonome, le problème de sa fondation logique et sa tendance à confondre théorie et méthodologie. Il avait aussi dénoncé la confusion existant entre thématisation de la traduction et réflexion sur la traduction. Rompu au raisonnement philosophique et pétri de culture gréco-latine, ce spécialiste de la philosophie allemande déployait une grande érudition dans son ouvrage, qui avait pour toile de fond les mythes d'Hermès et d'Apollon.

Cette fois-ci, Charles Le Blanc prolonge sa réflexion à partir des conclusions du Complexe d'Hermès. La mythologie grecque est remplacée par cinq contes et récits bien connus autour desquels il charpente son argumentation. Du jamais vu, sauf erreur, en traductologie. Ces contes et récits sont

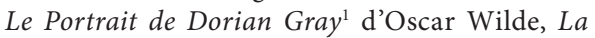
Reine des neiges ${ }^{2}$ d'Andersen, L'Apprenti sorcier ${ }^{3}$

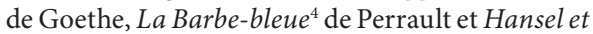
Gretel $^{5}$ de Grimm. Par des rapprochements analogiques avec ces œuvres, l'auteur décrit cinq grandes caractéristiques de la traduction et cinq périodes de l'art de traduire, de l'Antiquité au romantisme. Un extrait de la quatrième de couverture expose clairement l'utilisation originale qu'il fait de ces contes:

Comme le portrait de Dorian Gray, les traductions vieillissent alors que l'original conserve une éternelle jeunesse. Comme dans les morceaux du miroir magique brisé du conte d'Andersen, c'est le regard du lecteurtraducteur sur le texte qui en reconstruit le sens. Comme dans la ballade de Goethe, la multiplication des traductions rappelle celle des balais déchaînés par l'apprenti sorcier qu'est le traducteur: celui-ci doit pourtant reconnaître que l'auteur reste le seul maître. Comme dans le conte de Perrault, une œuvre littéraire est un château dont l'auteur, tel Barbe-bleue, tend le trousseau de clés au lecteur; ce que le texte deviendra une fois traduit dépend en grande partie de la clé que le traducteur utilisera. Enfin, tout traducteur part à la recherche du sens de l'œuvre, et comme Hansel et Grethel chez les frères Grimm, il espère bien revenir à la maison paternelle, c'est-à-dire à l'original. Mais s'il arrive qu'il s'égare, il peut aussi parvenir à des richesses insoupçonnées, comme les deux enfants découvrant la maison de pain d'épice. (Quatrième de couverture)

Dans ce nouvel essai, Le Blanc pose la question, qui peut paraître banale, mais qui ne l'est pas: «Qu'est-ce que la traduction?» Et il répond pertinemment: son histoire. Cette réponse implique 
que la figure du lecteur passe au premier plan. La traduction est à la fois une pratique ancrée dans l'Histoire et une maïeutique du sens à laquelle procède le traducteur-lecteur, notion clé de son essai. Vue sous cet angle, la traduction est une œuvre de substitution, une lecture écrite: "Une traduction n'est pas un original autrement, mais une lecture originale d'un texte qui témoigne du rapport entretenu entre un auteur et ce lecteur si particulier qu'est le traducteur» (p. 16-17). Tout comme «la beauté est dans les yeux de celui qui regarde» (Oscar Wilde), la traduction se crée à travers l'œil du traducteur-lecteur : « La traduction est le portrait d'une œuvre à travers un regard» (p. 93).

Le texte littéraire, à l'instar de toute traduction, est porteur d'une historicité, car il est porteur d'un usage de la langue à une époque donnée. Cette analyse pénétrante montre bien que le texte littéraire n'est pas clos, mais ouvert (contrairement au texte pragmatique) et qu'il faut sortir le discours sur la traduction des débats stériles sur la lettre ou l'esprit (faut-il traduire littéralement ou librement?).

La notion même de "traduction", écrit l'auteur, n'a pas tout à fait le même sens d'une époque à une autre, et cela parce qu'on ne lit pas les textes de la même façon selon le siècle où l'on se trouve. Comprendre comment on a traduit un texte, c'est comprendre comment on l'a lu, mais aussi quand cette lecture est intervenue. (p. 294)

Pour un historien de la traduction, ce constat a valeur d'axiome.

Contrairement à la plupart des traités antérieurs, de Cicéron à Berman, en passant par Leonardo Bruni et Dolet, cet essai ne cherche pas à dire ce que doit être (ou ne pas être) une traduction - combien de traités et de théories de la traduction s'apparentent à des méthodologies -, mais tente de cerner ce qu'est cette activité complexe et polymorphe. Pas plus que le premier essai, paru en 2009, Histoire naturelle de la traduction n'est un ensemble de règles à suivre, une vaine tentative de «codification» de la traduction.

Une théorie de la traduction exige une théorie de la lecture. On peut dire d'Histoire naturelle de la traduction ce qu'Annie Brisset a écrit au sujet du collectif codirigé par Le Blanc et Simonutti (2014) : «Il pose, dans une perspective historique, la question essentielle du cadre de référence qui informe l'interprétation d'un texte et sa reformulation dans un espace-temps donné» (Brisset 2019: 135). Cet énoncé résume bien le principe de lecture écriture que développe l'auteur.

Le Complexe et Histoire naturelle ont aussi en commun une dénonciation de cette sorte de mystique de la lettre qui tend à sacraliser l'original.
C'est pourquoi, dans un souci de changement de perspective, aux couples texte de départ / texte d'arrivée, texte source / texte cible, l'auteur préfère le binôme texte originel et texte originaire, comme on dit d'un immigrant qu'il est originaire de tel ou tel endroit. Pour le dire autrement, le texte originel est le pays d'origine du texte originaire. Il est dans la nature même d'une traduction de venir de quelque part, et ce quelque part est le texte d'origine. Toute traduction est forcément un texte dérivé, et cela n'est pas sans conséquence. L'auteur en fait la démonstration en cinq chapitres.

Dans le premier chapitre, il fait voir comment le sens est l'objet d'une construction, comme l'est un portrait par rapport à un modèle et que, par conséquent, une traduction-portrait n'est jamais identique au modèle. Il explique aussi l'effet du temps sur les traductions, qui toutes vieillissent, contrairement aux ouvres d'origine.

Le deuxième chapitre porte sur la construction du sens par le traducteur-lecteur qui le bâtit avec tout son être et sa culture. Le texte littéraire n'est pas que les mots le constituant, mais aussi le regard qui le lit: «Or la traduction est une tentative explicite de mise à jour de ce regard» (p. 18).

Le troisième chapitre est consacré à la subjectivité du traducteur. Si celui-ci possède un certain pouvoir, ce n'est jamais celui de l'auteur. C'est en partie parce que le texte traduit n'arrive jamais à se faire l'égal du texte d'origine que les retraductions sont proposées. Ce sont les multiples balais de l'apprenti sorcier.

Dans le quatrième chapitre, l'auteur montre que "si le sens est bien transmission, on assiste néanmoins à des ruptures de réception, ruptures qui peuvent s'expliquer historiquement [...]» (p. 19). Ce que devient l'œuvre traduite dépend, en partie, de la clé que le traducteur utilise.

Dans le cinquième et dernier chapitre, l'auteur réhabilite «le dynamisme des lectures “déviantes" ou, si l'on préfère, des lectures qui ne suivent pas entièrement l'intentio auctoris dans la reconstruction du sens de l'œuvre lue» (p. 19). La traduction a cette propriété d'assurer à une œuvre littéraire une postérité renouvelée.

Par une démonstration claire, serrée, méthodique et d'une rigueur exemplaire, Le Blanc renouvelle le discours sur la traduction en mettant le traducteur-lecteur au centre de sa réflexion. Le langage abscons est totalement absent de cette réflexion, et son essai, que je n'hésite pas à qualifier de brillant, se pare de qualités littéraires qui en agrémentent la lecture. Je ne doute pas un seul instant que cette contribution majeure trouvera un écho retentissant en traductologie et dans plusieurs autres domaines d'études.

Le titre Histoire naturelle de la traduction, qui évoque spontanément l'Histoire naturelle de 
Pline l'Ancien, appelle une explication. L'auteur justifie le choix de ce titre ainsi: dans l'ouvrage de Pline, la Nature est une force agissante et raisonnable qui forme le monde et insuffle une vie à toute chose. Cette idée, Le Blanc la transpose à la littérature en assignant au lecteur «une force motrice et rationnelle qui donne forme, sens et actualité à tout ce à quoi elle s'applique» (p. 20). Le lecteur est à la traduction ce que la Nature est à la vie. Chacun jugera de la pertinence de cette analogie.

Pour clore cette recension et donner un aperçu du style aphoristique de l'auteur (n'a-t-il pas traduit de l'allemand le recueil d'aphorismes, Le miroir de l'âme, de Lichtenberg [2012]?), j'ai pensé proposer un échantillon de quelques passages ayant la force de frappe des maximes et qui auraient pu figurer en bonne place dans mon florilège de citations (Delisle 2007/2017).

1) Une bonne partie de l'art du traducteur consiste à rendre un sens à travers des mots et non des mots à travers d'autres mots». (p. 11)

2) Le voyage d'un traducteur à travers une page blanche n'est pas moins périlleux que celui d'un auteur. (p. 23)

3) L'écrivain est devant tous les possibles. Le traducteur, devant tous les possibles qu'il lui est possible d'exprimer. (p. 23)

4) Une traduction n'est pas la reproduction de l'original, elle en est le symbole, car elle procède à sa substitution, elle "prend la place de" comme le fait couramment le symbole, ce qui est, du reste, sa fonction première. (p. 49)

5) Une traduction peut être fausse, jamais un original. (p. 61)

6) Une traduction est un texte qui porte en lui ses conditions de réfutabilité. (p. 62)

7) On juge l'original de façon critique; sa traduction de manière analytique. (p. 65)

8) Une traduction révèle moins un original qu'une façon circonstanciée de le lire. (p. 69)

9) Le déplacement du temps de l'œuvre dans celui de l'œuvre-traduite apparaît essentiel pour juger du travail du traducteur. (p. 71)

10) Tout effort de traduction cherche à exprimer, en plus de l'œuvre, la façon de la lire. (p. 74)

11) La traduction d'une œuvre ne sera jamais meilleure que la lecture qui en a été faite. (p. 100)

12) Les différentes traductions d'un même texte mettent en scène le conflit des lectures, elles orchestrent concrètement la pluralité des sens possibles. (p. 168)

13) Il ne suffit pas, pour lire, d'avoir des livres: il faut, pour lire, avoir lu. (p. 182)

14) Ut legis, ita vertis: comme tu lis, ainsi traduistu. (p. 222)

15) On ne le répétera jamais assez: la traductologie ne doit pas se fonder d'abord sur des concepts, mais sur des pratiques. (p. 234)
16) Ce qui était jugé fidèle autrefois ne l'est plus aujourd'hui. La fidélité n'est donc pas une qualité intrinsèque et un élément constitutif de l'art de traduire: elle n'est jamais que l'expression du préjugé d'une époque. (p. 239)

L'essai de Charles Le Blanc nourrit de façon originale la réflexion sur la traduction, en renouvelle les métaphores et tisse des relations insoupçonnées entre histoire, littérature, contes fantastiques, philosophie, études anciennes et traduction, tout en restant parfaitement cohérent, clair et lisible. Un régal pour l'esprit!

Jean Delisle

Université d'Ottawa, Ottawa, Canada

\section{NOTES}

1. Wilde, Oscar (1890): The Picture of Dorian Gray. Lippincott's Monthly Magazine. July:1-100.

2. Andersen, Hans Christian (1844): Snedronningen [La Reine des neiges]. In: Hans Christian Andersen. Nye Eventyr [Nouveaux contes]. Copenhague: C. A. Reitzel.

3. Goethe, Johann Wolfgang von (1797/1827): Der Zauberlehrling [L'apprenti sorcier]. In: Johann Wolfgang von Goethe. Goethe's Werke: vollständige Ausgabe letzter Hand [CEuvres de Goethe: édition finale et complète]. Stuttgart/Tubingue: J. G. Cotta, 217-220.

4. Perrault, Charles (1697/1867): La Barbebleue. In: Charles Perrault. Les contes de Perreault. Paris: J. Hetzel, 60-66.

5. Grimm, Jacob et Grimm, Wilhelm (1812): Hänsel und Gretel [Hansel et Gretel]. In: Jacob Grimm et Wilhelm Grimm. Kinder- und Hausmärchen [Contes de l'enfance et du foyer]. Berlin: Realfchulbuchhandlung, 49-58.

\section{RÉFÉRENCES}

Brisset, Annie (2019): La traductologie canadienne au fil des publications (1970-2017). TTR. 30(12) :121-148.

Delisle, Jean (2011): Un coup de critique dans la fourmilière traductologique. TTR. 24(1):235243.

Delisle, Jean (2007/2017): La traduction en citations: Florilège. $2^{\mathrm{e}}$ éd. Ottawa: Presses de l'Université d'Ottawa.

Le Blanc, Charles (2009): Le complexe d'Hermès. Regards philosophiques sur la traduction. Regards sur la traduction. Ottawa: Presses de l'Université d'Ottawa.

Le Blanc, Charles et Simonutti, Luisa (2014): Le masque de l'écriture: philosophie et traduction de la Renaissance aux Lumières. Genève/ Rome: Droz/CNR.

Lichtenberg, Georg Christoph (2012): Le miroir de l'âme. $3^{\mathrm{e}}$ éd. (Traduit de l'allemand par Charles Le Blanc) Paris: J. Corti. 